\title{
3D models related to the publication: Morphogenesis of the stomach during the human embryonic period
}

\section{$\operatorname{AUTHOR}(\mathrm{S})$ :}

Nako, Ami; Kaigai, Norihito; Shiraki, Naoto; Yamada, Shigehito; Uwabe, Chigako; Kose, Katsumi; Takakuwa, Tetsuya

\section{CITATION:}

Nako, Ami ...[et al]. 3D models related to the publication: Morphogenesis of the stomach during the human embryonic period. MorphoMuseuM 2015, 1(4): e3.

\section{ISSUE DATE:}

2015

URL:

http://hdl.handle.net/2433/216938

\section{RIGHT:}

(C) Copyright Tetsuya Takakuwa 2015; All 3D data presented on this website are licensed under a Creative Commons AttributionNonCommercial 4.0 International License. 


\title{
3D models related to the publication: Morphogenesis of the stomach during the human embryonic period
}

\author{
NAKO A ${ }^{1}$, KAIGAI ${ }^{1}$, SHIRAKI $\mathbf{N}^{1}$, YAMADA $\mathbf{S}^{1,2}$, UWABE $\mathrm{C}^{2}, \operatorname{KOSE~} \mathrm{K}^{3}$, TAKAKUWA $\mathbf{T}^{1^{*}}$ \\ ${ }^{1}$ Human Health Science, Graduate School of Medicine, Kyoto University, Kyoto 606-8507, Japan \\ ${ }^{2}$ Congenital Anomaly Research Center, Graduate School of Medicine, Kyoto University, Kyoto 606-8501, Japan \\ ${ }^{3}$ Institute of Applied Physics, University of Tsukuba, Tenoudai 1-1-1, Tsukuba, Ibaragi, 305-8573, Japan \\ * corresponding author: Dr. Tetsuya Takakuwa(tez@hs.med.kyoto-u.ac.jp)
}

Abstract: The present 3D Dataset contains the 3D models analyzed in: Kaigai N et al. Morphogenesis and three-dimensional movement of the stomach during the human embryonic period, Anat Rec (Hoboken). 2014 May; 297(5):791-797. doi: 10.1002/ ar.22833.

Key words: human stomach, human embryo, magnetic resonance imaging, three-dimensional reconstruction

Submitted 12.11.2015, Accepted 13.11.2015. doi: 10.18563/m3.1.4.e3

(C) Copyright Tetsuya Takakuwa 2015

\section{SPECIMEN LIST}

The morphogenesis of the human stomach was visualized using images derived from human embryo specimens between Carnegie stage (CS) 16 and CS23 from the Kyoto Collection, which were acquired with a magnetic resonance microscope equipped with a 2.35-T superconducting magnet.

\begin{tabular}{|c|c|c|}
\hline \multicolumn{1}{|c|}{ Model ids } & Taxon & Developmental stage (Carnegie stage (CS)) \\
\hline M3\#56_KC-CS16STM27159 & Homo sapiens & CS 16 \\
\hline M3\#57_KC-CS17STM20383 & Homo sapiens & CS 17 18 \\
\hline \hline M3\#58_KC-CS18STM21807 & Homo sapiens & CS 19 \\
\hline M3\#59_KC-CS19STM17998 & Homo sapiens & CS 20 \\
\hline M3\#60_KC-CS20STM20785 & Homo sapiens & CS 21 \\
\hline M3\#61_KC-CS21STM24728 & Homo sapiens & CS 22 \\
\hline \hline M3\#62_KC-CS22STM26438 & Homo sapiens & CS 23 \\
\hline \hline M3\#63_KC-CS23STM20018 & Homo sapiens & \\
\hline \hline
\end{tabular}

\section{METHODS}

Well-preserved human embryos between Carnegie stage (CS) 16 and the CS23 (approximately 6-8 weeks after fertilization) were selected from Kyoto Collection for MR microscopic imaging (Nishimura et al, 1968; Shiota et al, 2007; O'Rahilly \& Müller, 1987).

The MR images of the embryos were acquired using a superparallel MR microscope developed with a $2.35 \mathrm{~T}$ horizontal bore $(40 \mathrm{~cm})$ superconducting magnet (Matsuda et al., 2007). MRI images from selected embryos were analyzed precisely as serial 2D and reconstructed $3 \mathrm{D}$ images. The structure of the stomach was reconstructed in all samples using Amira software version 5.4.5 (Visage Imaging; Berlin, Germany). The 3D surface models were then processed with ISE-MeshTools
(Lebrun, 2014); each model was orientated and labelled using this software. All labels were provided in .flg format. The 3D surface models were also provided in .ply format, and could therefore be opened with a wider range of freeware.

This study was approved by the Committee of Medical Ethics of Kyoto University Graduate School of Medicine, Kyoto, Japan (E986).

\section{ACKNOWLEDGEMENTS}

This study was supported by Grant Nos. 25461642, 24119002, 26220004, 15H01119, 15K08134, 15H05270, 15H01121, and $15 \mathrm{~K} 15014$ from the Japan Society for the Promotion of Science 
MMM JOURNAL VOL.1 (4)-e3

16

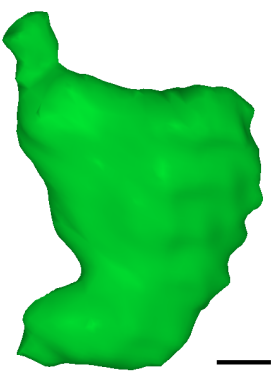

19

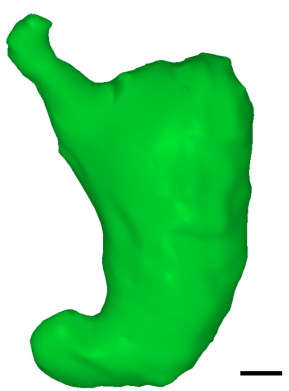

23

\section{cardiac incisure}

(His angle)

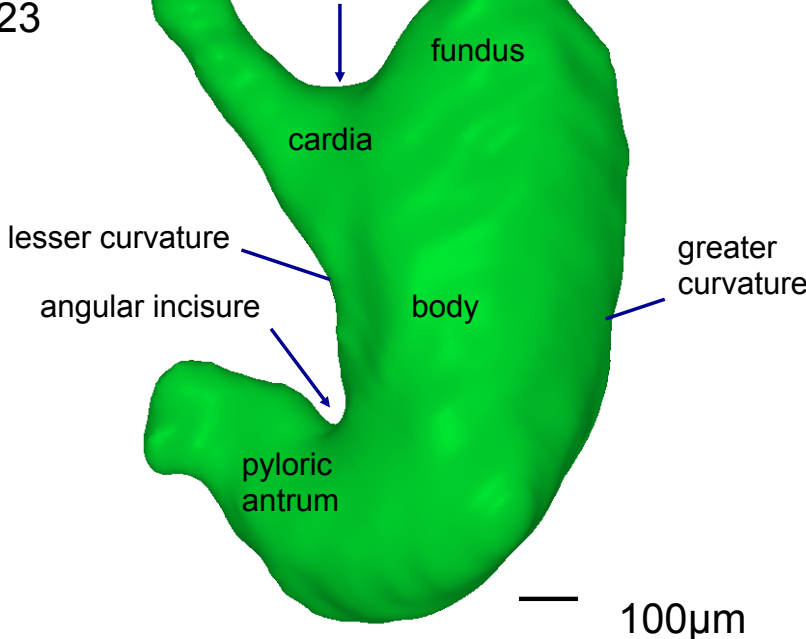

Figure 1: Representative images of the stomach at Carnegie stage (CS)16, CS 19 , and CS23.

\section{BIBLIOGRAPHY}

Kaigai, N., Nako, A., Yamada, S., Uwabe, C., Kose, K., Takakuwa, T., 2014. Morphogenesis and threedimensional movement of the stomach during the human embryonic period. Anat Rec (Hoboken) 297, 791-797. doi: 10.1002/ar.22833.

Lebrun, R., 2014. ISE-MeshTools, a 3D interactive fossil reconstruction freeware. 12th Annual Meeting of EAVP, Torino, Italy.

Matsuda, Y., Ono, S., Otake, Y., Handa, S., Kose, K., Haishi, T., Yamada, S., Uwabe, C., Shiota, K., 2007. Imaging of a large collection of human embryo using a super-parallel MR microscope. Magn Reson Med Sci 6, 139-146. doi: $\underline{10.2463 / \mathrm{mrms} .6 .139}$

Nishimura, H., Takano, K., Tanimura, T., Yasuda, M., 1968. Normal and abnormal development of human embryos: first report of the analysis of 1,213 intact embryos. Teratology 1, 281-290. doi: 10.1002/tera.1420010306

O'Rahilly, R., Müller, F., 1987. Developmental stages in human embryos: including a revision of Streeter's Horizons and a survey of the Carnegie Collection. Washington, D.C.: Carnegie Institution of Washington.

Shiota, K., Yamada, S., Nakatsu-Komatsu, T., Uwabe, C., Kose, K., Matsuda, Y., Haishi, T., Mizuta, S., Matsuda, T., 2007. Visualization of human prenatal development by magnetic resonance imaging (MRI). Am J Med Genet A 143A, 3121-3126. doi: 10.1002/ajmg.a.31994 